\title{
Disseminated Histoplasmosis in an Elderly Man Presented with Fever, Weight loss, Abdominal Pain and Haemoptysis - A Case Report with Literature Review
}

\author{
MD ROBED AMIN ${ }^{1}$, FARZANA SHUMI ${ }^{2}$, HASI BUDDIN KHAN ${ }^{2}$, SYED AHMED ABDULLAH ${ }^{2}$, SHAFIUL ALAM ${ }^{3}$, AHMED \\ RIYAD HUSSAIN ${ }^{4}$, MAKAHHAR ${ }^{5}$
}

\begin{abstract}
An elderly patient presented with prolonged fever, gross weight loss, recurrent haemoptysis and abdominal pain. He had a background history of adrenal tuberculosis with completion of treatment without any obvious improvement. Clinically he was diagnosed as a case of adrenocotical insufficiency. Evaluation including histopathology revealed the diagnosis as disseminated histoplasmosis involving adrenal gland and lungs. The disease is a rarity without any underneath immunosuppression and hence high index of suspicion with appropriate steps for investigation is the key to achieve a diagnosis of disseminated histoplasmosis in Bangladesh.
\end{abstract}

Keyword: Disseminated Histoplasmosis, Mycosis, Immunosuppression, Adrenal Gland, Bangladesh.

\section{Introduction:}

Histoplasmosis caused by Histoplasma capsulatum var capsulatum is rare in Bangladesh. There is no defined endemic area in this zone of South East Asia to have hisplasmosis occurring regularly.Exposure to H. capsulatum is exceedingly common for persons living within areas of endemicity, but symptomatic infection is uncommon. ${ }^{1}$ The vast majority of infected persons have either no symptoms or a very mild illness that is never recognized as being histoplasmosis. Hematogenous dissemination is common in the early stages of infection with $\mathrm{H}$ capsulatum, but almost all people in whom this occurs remain asymptomatic.

Symptomatic acute disseminated infection occurs almost entirely in those who are immunosuppressed. ${ }^{2,}$, ,4-12

The symptoms of disseminated histoplasmosis include fever, malaise, anorexia, and weight loss. Physical examination will often show hepatosplenomegaly, lymphadenopathy, pallor and petechiae if pancytopenia is present, and, in some patients, mucous membrane ulcerations as well as skin ulcers, nodules, or molluscum-like papules. ${ }^{13,14}$ Laboratory investigation usually showed high ESR, CRP, Lactate Dehydrogenase, Ferritin which although are not specific but commonly to be found in appropriate setting. Chest radiographs may be normal or show a diffuse reticulonodular infiltrate. For patients who are seriously ill, tissue biopsy for histopathologic evaluation for fungi is a rapid means to establish a diagnosis of histoplasmosis. ${ }^{15}$

In disseminated histoplasmosis virtually any organ can be involved but symptomatic disease is rare at some sites. Adrenal gland is the site where disease is uncommon and even if present, it mostly showed unusual features and so difficult to diagnose. Here is a case report of a patient suffering from disseminated histoplasmosis presenting with adrenal gland involvement who suffered few years with multiple consultation before confirmation of diagnosis of disseminated histoplasmosis.

\section{Case Report:}

A 65 year old school teacher presented with fever for three months, weight loss, anorexia, weakness and abdominal pain for same duration. The fever was low grade, intermittent, with evening rise and not associated with chills and rigor but subsided by sweating spontaneously. He also had anorexia, fatiguability and generalized weakness during this whole three months and reported to have lost of about $14 \mathrm{~kg}$ of weight. He also experienced two episodes of haemoptysis for two within last three months. He also complaints of intermittent dull aching upper abdominal pain . There was occasional nausea and vomiting which was not related to the pain. He is a smoker with smoking habit of 40 pack yr. For these complaints he took advice in home and abroad from

1. Assistant Professor, Department of Medicine, Dhaka Medical College.

2. Honorary Medical Officer, Department of Medicine, Dhaka Medical College

3. Indoor Medical Officer, Department of Medicine, Dhaka Medical College

4. Assistant Registrar, Department of Medicine, Dhaka Medical College

5. Professor, Department of Medicine, Dhaka Medical College.

Correspondence: Dr.Md. Robed Amin, Assistant Professor of Medicine, Department of Medicine, Dhaka Medical College, Bangladesh. e.mail: robedamin@yahoo.com 


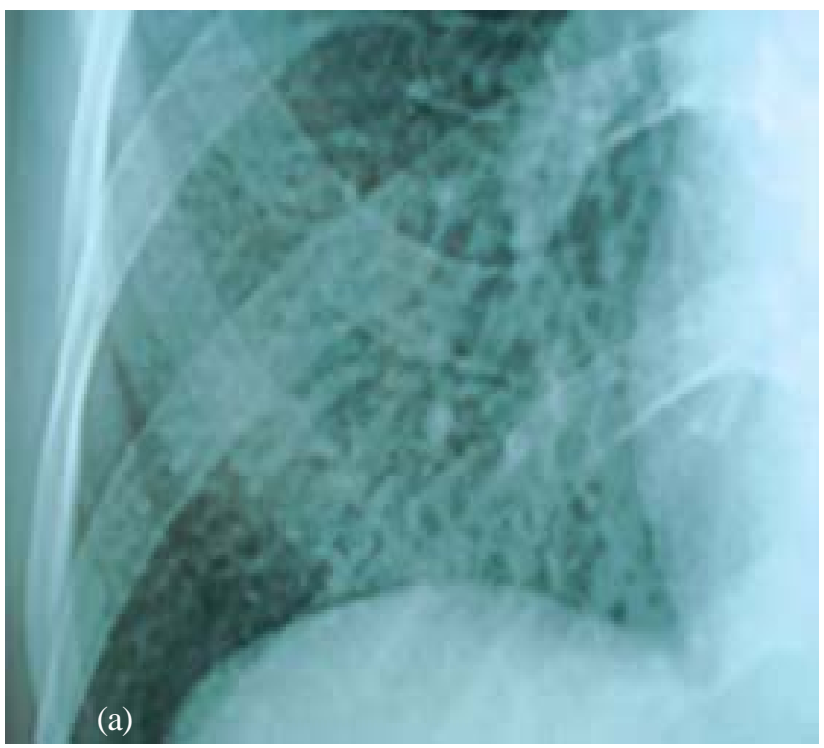

Fig.-1: (a) CXR showing reticulonodular shadow in and

different consultants. First, he was diagnosed as a case of liver abscess and was treated with injectable antibiotics and completely recovered. He was also diagnosed as a case of adrenal tuberculosis based on clinical symptoms and signs and tissue diagnosis for which he received anti tubercular chemotherapy for 8 months. During and after receiving antitubercular chemotherapy there was no significant improvement of his symptoms; rather he experienced recurrent cough with occasional oral ulcer, proctalgia, body ache and visual disturbance. He has history of total 8 units of blood transfusion on two occasions for his anaemia and weakness. On examination he was found ill looking with below average body built (BMI=17.92), febrile (101 degree F) .His blood pressure was $100 / 70 \mathrm{~mm}$ of $\mathrm{Hg}$ in supine position and 90/70 $\mathrm{mm}$ of $\mathrm{Hg}$ in erect posture. There was

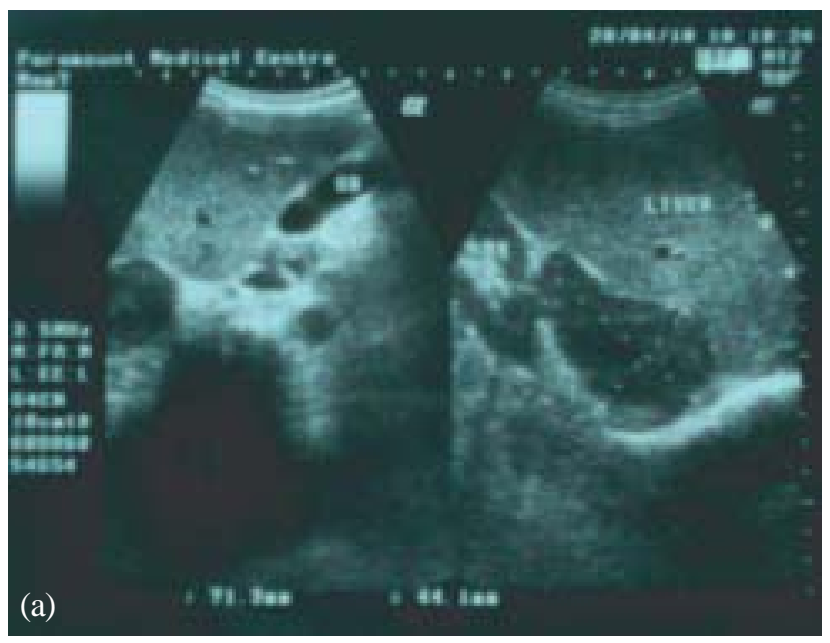

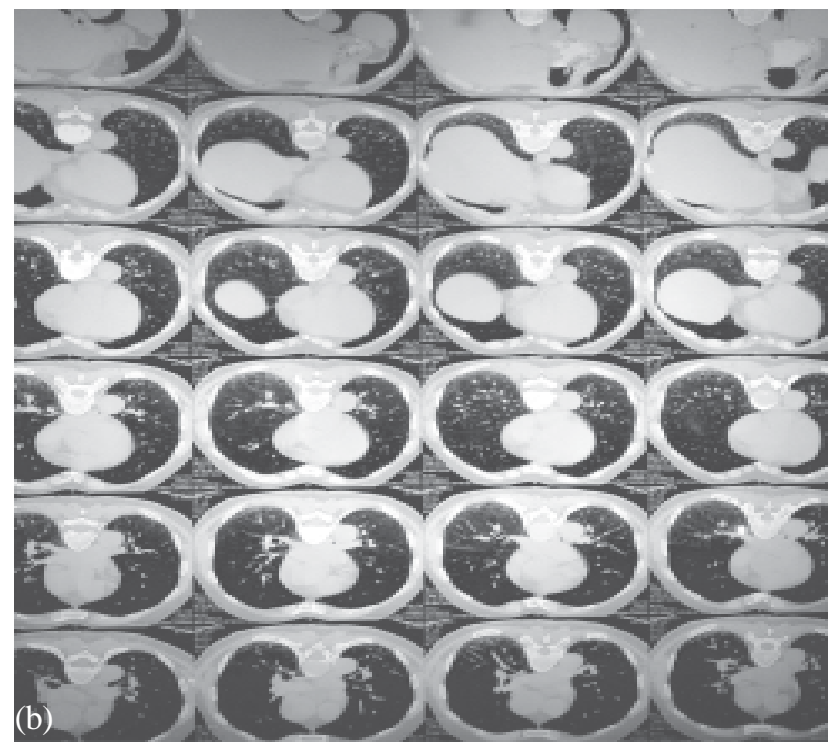

(b) CT chest revealed Fibrosis and bronchiectasis

palmer erythema. He had white patches in the oral cavity, liver was palpable $8 \mathrm{~cm}$ from costal margin but hepatic span was $14 \mathrm{~cm}$, inspiratory crackles on lower zone of both lung field was present. Other systemic examinations were normal. Investigations revealed his $\mathrm{Hb} \%: 12.9 \mathrm{mg} / \mathrm{dl}, \mathrm{TC}: 9600, \mathrm{DC}: \mathrm{N}-$ 70\%,L-22\%,M-06\%,E-02\% ,normocytic normochromic anaemia was found in his PBF and his ESR was $14 \mathrm{~mm}$ in the first hour.

Chest X-Ray revealed reticulo nodular opacities with honeycomb appearance visible on both lower zones but more on the left side. CT Chest suggestive of old PT with fibrotic and bronchiectatic changes in the left lung upper lobe. Emphysematous bilateral lung fields, mild and generalized dilatation of major airways were also observed.

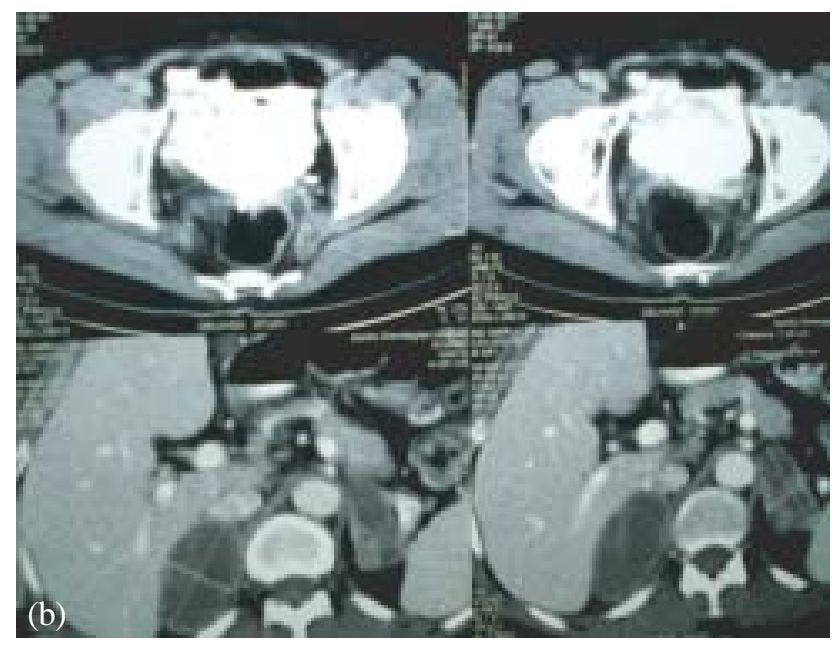

Fig.-2: (a) USG abdomen showed bilateral (b): His LFT was CT abdomen showed solid and central 

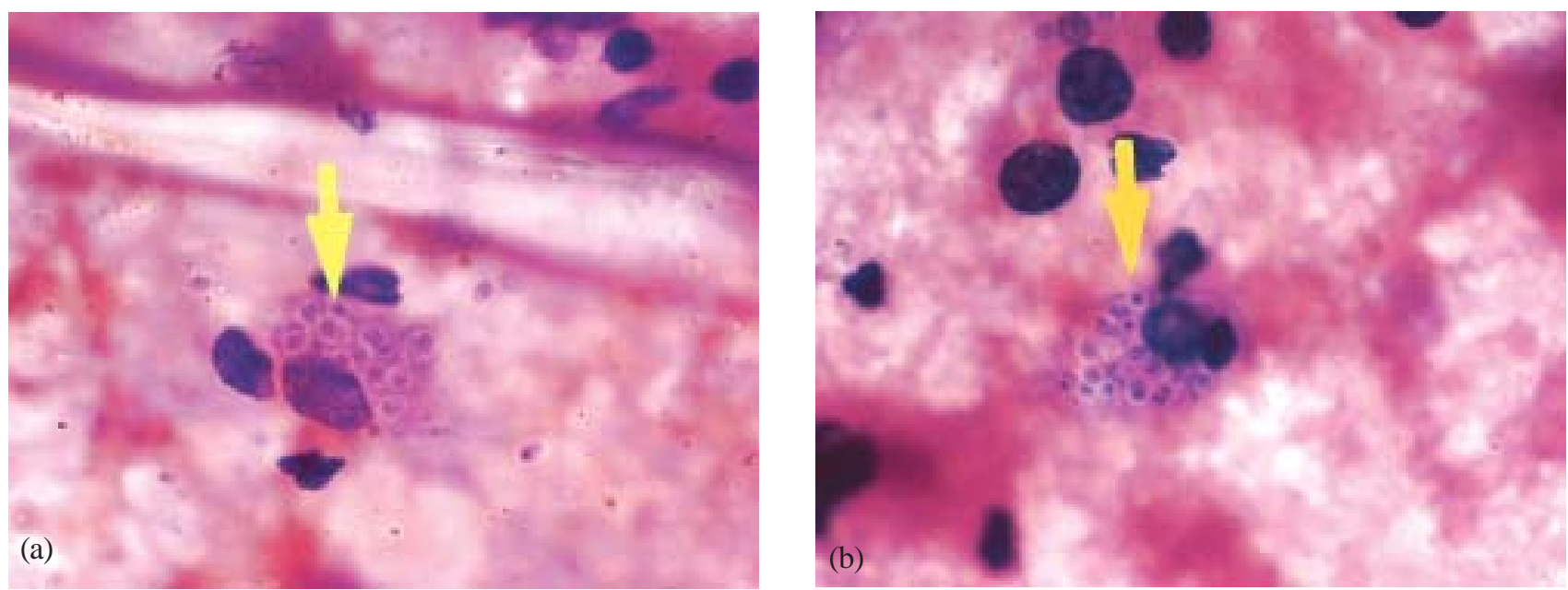

Fig.-3: (a) and (b): FNA of adrenal mass shows histiocyte contain cytoplasmic protozoa having round to cresentic nucleus and perinuclear halo. (CProf Md Kamal

Ultrasonography of whole abdomen revealed bilateral suprarenal masses (one hypoechoeic mass measuring about $71 \mathrm{X} 44 \mathrm{~mm}$ in size seen in right suprarenal region and another $40 \mathrm{X} 28$ in the left). CT abdomen revealed well-defined solid and central necrotic masses in bilateral adrenal glands larger on the right side. Other investigations done which revealed S .bilirubin-1.0 mg/dl S. Alkaline phosphatase: 592 U/L(20-140 IU/L)SGOT: 83.2 (07-56). SGPT: 81.9 (05-40). S. albumin-3.8 g/dl Total protein-7.5 g/dl, Serum Globulin-3.7 g/dl. S.Ferritin-752 ng/ml. Upper GI endoscopy was normal. Sputum for AFB for 3 samples were negative.

The lung function tests were within normal limit. FNAC from right adrenal mass was done and smears reveal blood, lymphocytes and scattered histiocyte.

The histiocytes contain cytoplasmic protozoa having round to crescentic nucleus and perinuclear halo. No malignant cell was seen. The bone marrow examination was normal. Rapid ACTH stimulation test reveals Cortisol 0 min: 945.67 nmol/l ( Normal value>1065 ) Cortisol 30 min:1036.37 Cortisol 60 min:931.13 which were consistent with partial adrenocortical insufficiency. VDRL test was negative, HIV

I/II was negative and HBsAg and Anti HCV were also negative. Blood culture and sensitivity showed no growth. The serological test with antigen for histoplasma and antibody test for histoplasmosis could not done due to lack of facility. The clinical profile and investigation revealed the diagnosis of disseminated histoplasmosis with partial adrenocortical failure.

\section{Discussion:}

Histoplasmosis is the most common endemic mycosis causing human infection first described a little over a century ago by an American physician, Samuel Darling. Histoplasmosis is caused by histoplasma capsulatum which is a dimorphic fungus that exists as a mold in the environment and as a yeast in tissues at $37 \mathrm{C} .{ }^{15}$ There are two varieties of H. capsulatum that are pathogenic to humans, H. capsulatum var. capsulatum and $\mathrm{H}$. capsulatum var. duboisii. ${ }^{1}$ The ability to contain infection is almost entirely mediated by cellmediated immunity. So peoples who have defined intrinsic or extrinsic defects in cell mediated immunity is more prone to develop disseminated diseases. These organisms are held in check by the immune response but are not completely killed, and thus, reactivation of infection years later is possible. ${ }^{16,17,18}$ The major risk factors that portend an increased risk for disseminated infection with $\mathrm{H}$ capsulatum are exposure to the organism as an infant before cell-mediated immunity is adequate to handle intracellular pathogens corticosteroids and other immunosuppressive agents given for various conditions, AIDS with CD4 cells less than 150/ $\mathrm{mL}$ hematologic malignancies, and solid organ transplantation. 5,8,15,19-23 With increasing use of tumor necrosis factor (TNF) antagonists in many rheumatological disorders, this form of therapy has emerged as a major risk factor for histoplasmosis. ${ }^{9,10,11}$ Interestingly in our patient there was no specific travel to an endemic area known to have histoplasmosis in abundance nor he took any immunosuppresive drugs. Later attempts have been done in investigation to find out immunosuppresive states which was negative in this case. His job was not also consistent with the easy exposure to deep fungus. He was diagnosed as a case of adrenal tuberculosis which itself can lead to 
reduced his immunity by reducing endogenous steroid and thus leads to development of histoplasmosis but the source of infecting agent still remain obscured. Chronic progressive disseminated histoplasmosis has the indolent course and is uniformly fatal if not treated. Our case the scenario is consistent with the statement and possibly he is a case of Chronic disseminated histoplasmosis and the total illness duration and the gradual severity of illness supports it.

The most common clinical manifestations are chills, fever, malaise, anorexia, and weight loss. ${ }^{13}$ Several unique manifestations occur more frequently with histoplasmosis than with other disseminated fungal infections. This includes Addison's disease, which occurs when there is extensive destruction of both adrenal glands by the infection. The patient exhibits fever, malaise, fatigue, orthostatic hypotension, nausea, and vomiting. ${ }^{24,25} \mathrm{CT}$ scan shows markedly enlarged adrenals, often with necrosis in the central area. ${ }^{26}$ In our case, the picture is consistent with the features of adrenal failure that developed gradually and investigations also revealed partial deficiency in ACTH stimulation test. Partial deficiency may be reason why he did not have postural hypotension or electrolyte abnormalities but his symptoms were gradually progressing. We found white patches in oral cavity and ulceration. Liver was palpabe but no splenomegaly was found. This indicates the variability which is a consistent features in dissemination. Increased erythrocyte sedimentation rate, pancytopenia, elevated alkaline phosphatase, lactate dehydrogenase, ferritin and diffuse pulmonary infiltrates reflect widespread involvement. This form of histoplasmosis, although chronic, is fatal if not treated. The investigations profile was consistent in our patient although these tests are not specific. In a highly suspected cases these test have enormous importance in diagnosis. Chest X-ray and CT chest shows reticulonodular shadows in our patient which were misinterpreted by many physician as part of Chronic obstructive pulmonary diseases . The persistent symptoms of respiratory system and non response to bronchodilators is also a clue to an underlying fungal infection in this patient. Another interesting features in this case report were history of tuberculosis in adrenal gland two years back. At that time FNAC of adrenal gland showed caseous necrosis with granulomatous inflammation. The Anti tubercular chemotherapy although was prescribed and took by the patient for 8 months, there was hardly any improvement in symptoms. Although he was diagnosed as adrenal tuberculosis but as there was no adrenal insufficiency, he was not prescribed with steroids. The histological features of tuberculosis can also be the same in histoplasmosis and may be the initial diagnosis of adrenal tuberculosis was actually due to histoplasmosis of adrenal gland. The slowness of destruction of adrenal gland was really surprising observation in this case and no remarkable skin hyperpigmentation was seen.

The tuberculate macroconidia are characteristic of $\mathrm{H}$ capsulatum, but identification should be verified with the use of DNA probe for confirmation which is also a confirmatory test with high specificity. In our case we could not culture the organism from tissue due to unavailability of culture medium. The DNA probe is not possible with current diagnostic facilities of Bangladesh. Measurement of antibodies against $\mathrm{H}$. capsulatumis accomplished by complement fixation (CF) and immunodiffusion (ID) assays. ${ }^{23,27}$ Antigen and Antibody tests are most useful for diagnosis and for prognosis in patients who have chronic progressive disseminated histoplasmosis, both CF and ID tests are useful . Diagnosis should not be based on a urine antigen test alone. It is recommended that urine histoplasma antigen levels be used as a guide to follow the course of a patient's illness. ${ }^{27,}{ }^{28}$ Due to unavailability of antigen and antibody tests in Bangladesh, we could not perform these tests in our patient. Patients who are not severely ill can be treated with oral itraconazole, $200 \mathrm{mg}$ twice daily or second line fluconazole, the dosage of which is 400 to $800 \mathrm{mg}$ daily. ${ }^{29,30}$ Patients with severe disseminated infection should be treated initially with amphotericin $B$ at a dosage of 0.7 to $1 \mathrm{mg} / \mathrm{kg}$ daily or a lipid formulation of amphotericin B at a dosage of 3 to $5 \mathrm{mg} / \mathrm{kg}$ daily. Infectious Diseases Society of America (IDSA) guidelines recommend 6 to 18 months total. In our patient we started the treatment with itraconazole. But unfortunately the patient couldn't tolerate the drug. Then intravenous amphotericin B was started as liposomal amphotericin B is not available in Bangladesh. But during the infusion, the patient developed fever and chest pain followed by collapse. He was rescued with adrenaline and steroids as per protocol of anaphylaxis management. An ECG was done and he was diagnosed as acute coronary syndrome having acute anterior myocardial infarction. He was treated then in CCU for this emergency. He recovered from the incident and after two weeks was started with fluconazole which he tolerated well. His general condition improved within a week, blood pressure rising upto 110/70 $\mathrm{mm} \mathrm{Hg}$. He was also treated with steroids due to adrenal insufficiency. On first month follow up his symptoms relieved about eighty percent and now he is on regular follow up.

\section{Conclusion:}

High index of suspicion is needed to diagnose the disseminated histoplasmosis infections. Bangladesh may be experiencing increased load of opportunistic fungal infections. The high clinical suspicion is important to clinch the diagnosis. It is essential to set up extensive laboratory 
facilities in Bangladesh to confirm and follow up the histoplasmosis cases.

\section{Conflict of Interest: None}

\section{References:}

1. Kauffman, C. A. Histoplasmosis. In W. E. Dismukes, P. G. Pappas, and J. D. Sobel (ed.), Clinical mycology. Oxford University Press, New York ;NY. 2003 p. 285-298

2. Kauffman CA. Endemic mycoses in patients with hematologic malignancies. Semin Respir Infect 2002; 17:106-12

3. McKinsey DS, Spiegel RA, Hutwagner L, et al. Prospective study of histoplasmosis in patientsinfected with human immunodeficiency virus: incidence,risk factors, and pathophysiology. Clin Infect Dis 1997; 24:1195-203

4. Odio CM, Navarrete M, Carrillo JM, et al. Disseminated histoplasmosis in infants. Pediatr Infect Dis J 1999; 18:1065-8

5. Couppie P, Sobesky M, Aznar C, et al. Histoplasmosis and acquired immunodeficiency syndrome:a study of prognostic factors. Clin Infect Dis 2004; 38:134-8

6. Assi MA, Sandid MS, Baddour LM, et al. Systemic histoplasmosis. A 15-year retrospective institutional review of 111 patients. Medicine (Baltimore) 2007; 86:162-9

7. Kauffman CA. Endemic mycoses after hematopoietic stem cell or solid organ transplantation. In:Bowden RA, Ljungman P, Paya CV, editors. Transplant infections. 2nd edition. Philadelphia: Lippincott Williams \& Wilkins; 2003. p. 524-34

8. Freifeld AG, Iwen PC, Lesiak BL, et al. Histoplasmosis in solid organ transplant recipients at a large midwestern university transplant center. Transpl Infect Dis 2005; 7:10915

9. Crum NF, Lederman ER, Wallace MR. Infections associated with tumor necrosis factor-alpha antagonists. Medicine (Baltimore) 2005; 84:291-302

10. Wood KL, Hage CA, Knox KS, et al. Histoplasmosis after treatment with anti-TNF-(alpha) therapy. Am J Respir Crit Care Med 2003;167:1279-82

11. Lee J-H, Slifman NR, Gershon SK, et al. Life threatening histoplasmosis complicating immunotherapy with tumor necrosis factor a antagonists infliximab and etanercept. Arthritis Rheum 2002; 46:2565-70

12. Limaye AP, Connolly PA, Sagar M, et al. Transmission of Histoplasma capsulatum by organ transplantation. N Engl J Med 2000; 343:1163-6

13. Goodwin, R. A., Jr., and J. L. Shapiro et al. Disseminated histoplasmosis: clinical and pathological correlations. Medicine (Baltimore) 1980; 59:1-33

14. Reddy, P., D. F. Gorelick, C. A. Brasher, and H. Larsh. Progressive disseminated histoplasmosis as seen in adults. Am. J. Med 1970; 48:629-636
15. Kauffman CA. Histoplasmosis: a clinical and laboratory update. Clin Microbiol Rev 2007; 20:115-32

16. Davies, S. F., M. Khan, and G. A. Sarosi. Disseminated histoplasmosis in immunologically suppressed patients. Am. J. Med. 1978; 64:94-100

17. Kauffman, C.A., K.S. Israel, J.W. Smith et al.. Histoplasmosis in immunosuppressed patients. Am. J. Med. 1978; 64:923-932

18. Mandell, W, D. M. Goldberg, H. C. Neu. Histoplasmosis in patients with the acquired immune deficiency syndrome. Am. J. Med. 1986; 81: 974-978

19. Odio CM, Navarrete M, Carrillo JM, et al. Disseminated histoplasmosis in infants. Pediatr Infect DisJ 1999; 18: 1065-8

20. Assi MA, Sandid MS, Baddour LM, et al. Systemic histoplasmosis. A 15-year retrospective institutional review of 111 patients. Medicine (Baltimore) 2007;86:162-9

21. Kauffman CA. Endemic mycoses after hematopoietic stem cell or solid organ transplantation. In: Bowden RA, Ljungman P, Paya CV, editors. Transplant infections. 2nd edition. Philadelphia: Lippincott Williams \& Wilkins; 2003. p. 524-34

22. McKinsey DS, Spiegel RA, Hutwagner L, et al. Prospective study of histoplasmosis in patients infected with human immunodeficiency virus: incidence, risk factors, and pathophysiology. Clin Infect Dis 1997; 24:1195-203

23. Kauffman CA. Endemic mycoses in patients with hematologic malignancies. Semin Respir Infect 2002; 17:10612

24. Sarosi G. A., D. W. Voth, B. A. Dahl, I. L. Doto, and F. E. Tosh. Disseminated histoplasmosis: results of long-term follow-up. Ann. Intern. Med.1971; 75:511-516

25. Smith, J. W., and J. P. Utz. Progressive disseminated histoplasmosis, a prospective study of 26 patients. Ann. Intern. Med. 1972; 76:557-565

26. Wilson, D. A., H. G. Muchmore, R. G. Tisda, A. Fahmy, and J. V. Pitha. Histoplasmosis of the adrenal glands studied by CT. Radiology 1984 ;150: 779-783

27. W heat LJ. Improvements in diagnosis of histoplasmosis. Expert Opin Biol Ther 2006; 6:1207-21

28. Wheat LJ, Connolly-Stringfield P, Kohler RB, et al. Histoplasma capsulatum polysaccharide antigen detection in the diagnosis and management of disseminated histoplasmosis in patients with acquired immunodeficiency syndrome. Am J Med1989; 89:396-400

29. Dismukes W. E , R. W. Bradsher, Jr., G. C. Cloud et al. and the NIAID Mycoses Study Group. Itraconazole therapy for blastomycosis and histoplasmosis. Am. J. Med. 1992; 93:489-497

30. Wheat, J., R. Hafner, A. H. Korzun, M. T. Limjoco, P. Spencer, R. A. Larsen, G. F. M. Hecht, W. Powderly, and the AIDS Clinical Trial Group. Itraconazole treatment of disseminated histoplasmosis in patients with the acquired immunodeficiency syndrome. Am. J. Med. 1995; 98:336-342 